\title{
Lacking hypoxia-mediated downregulation of E-cadherin in cancers of the uterine cervix
}

\author{
A Mayer ${ }^{\star}, 1$, M Höckel $^{2}$, N Schlischewsky ${ }^{1}$, H Schmidberger $^{1}$, L-C Horn ${ }^{3}$ and P Vaupel ${ }^{1}$ \\ ${ }^{1}$ Department of Radiooncology and Radiotherapy, University Medical Center, Mainz, Germany; ${ }^{2}$ Department of Gynaecology and \\ Obstetrics, University Medical Center, Leipzig, Germany and ${ }^{3}$ Department of Pathology, University Medical Center, Leipzig, \\ Germany
}

Background: Experimental studies have established a causal connection between tumour hypoxia, hypoxia-associated proteome changes and downregulation of E-cadherin, the final common pathway of epithelial-to-mesenchymal transition (EMT). Our study aimed at elucidating the interrelationship of these processes in cancers of the uterine cervix in vivo.

Methods: Tumour oxygenation was assessed in 48 squamous cell carcinomas (SCC) of the uterine cervix using polarographic needle electrodes. The expression pattern of E-cadherin was investigated by immunohistochemistry and western blotting, and was compared with that of the hypoxia-inducible proteins glucose transporter (GLUT)-1 and carbonic anhydrase (CA) IX in biopsy specimens of the oxygenation measurement tracks.

Results: The majority of cervical cancers (52\%) were E-cadherin positive, with a complete absence of the antigen in only $10 \%$ of the tumours. No correlation was found between the level of E-cadherin expression and the oxygenation status (mean $\mathrm{pO}_{2}$, median $\mathrm{pO}_{2}$ and hypoxic fractions). In patients showing partial expression of E-cadherin (38\%), staining was not preferentially diminished in GLUT-1- or CA IX-positive areas, and loss of E-cadherin occurred independently of tumour cell scattering.

Conclusion: Our data provide no evidence in favour of a hypoxia-induced EMT as a mechanistic basis of cervical cancer invasiveness.

Direct measurements of the oxygenation status using microelectrodes carried out in patients with cancers of the uterine cervix, the head and neck region and in soft tissue sarcomas have shown that critically lowered oxygen levels (hypoxia) within the tumour tissue are associated with a poor prognosis (Höckel et al, 1993, 1996, 1998; Brizel et al, 1996; Nordsmark et al, 2005). In one study on cervical cancer, this correlation with outcome was independent of the choice of treatment modality (Höckel et al, 1996), a finding that has indicated that tumour hypoxia may be a significant factor driving malignant progression (Höckel et al, 1996; Vaupel et al, 2004). With regard to the possible mechanisms underlying this phenomenon, hypoxia-induced proteome changes, which are coordinated primarily by the hypoxia-inducible factor (HIF)-1, may be of pivotal importance. This transcription factor is a heterodimeric protein whose $\alpha$-subunit (HIF-1 $\alpha$ ) is directly regulated by oxygen availability (Wang et al, 1995). Under normoxic conditions, HIF- $1 \alpha$ is modified by prolyl hydroxylase domain (PHD) enzymes and subsequently degraded via the proteasome, keeping its protein levels very low. Hypoxia interrupts this process and leads to its stabilisation, nuclear translocation and binding to its dimerisation partner HIF-1ß (Wenger, 2002). HIF-1 then activates the transcription of more than 800 genes (Semenza, 2012). Cellular processes driven by HIF-1 through this large-scale shift in gene expression are increasingly viewed as key mediators of hypoxia-associated malignant progression. This idea is supported by numerous studies that have shown clear correlations between elevated levels of hypoxia-inducible proteins and a poor patient prognosis (Vaupel and Mayer, 2007). Hypoxia-inducible factor -1mediated mechanisms include the metabolic adaptation ('reprogramming') to hypoxic, energy-deprived conditions, enabling

*Correspondence: Dr med A Mayer; E-mail: arnmayer@uni-mainz.de

This manuscript partly contains results from the Dr med. thesis of Nadja Schlischewsky.

Received 15 October 2012; accepted 27 November 2012;

published online 15 January 2013

(C) 2013 Cancer Research UK. All rights reserved 0007-0920/13

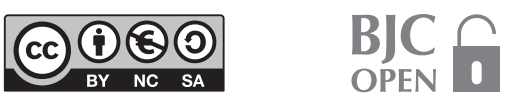


cancer cells to survive in hypoxic niches, for example by the upregulation of glucose influx via glucose transporter (GLUT)-1 and proton efflux by a mechanism involving membranous carbonic anhydrase (CA) IX. Owing to their longer half-lives and greater protein abundance compared with HIF- $1 \alpha$, both proteins have been used as surrogate markers for the activation of the hypoxic response in numerous studies.

Hypoxia-associated proteome changes have also repeatedly been connected to increased cancer cell invasion and metastasis - core traits of malignancy - by means of the induction of epithelial-tomesenchymal transitions (EMTs). As the term implies, this complex phenotypic transformation comprises the loss of epithelial and acquisition of mesenchymal characteristics, including an enhanced motility and local invasiveness associated with singlecell 'scattering'. Abolition of the tight cohesion found in normal epithelia absolutely requires the dissolution of mechanically stable physiological cell-to-cell contacts, in which the protein E-cadherin has a key role. Several in vivo and in vitro studies of tumours and tumour cells of different origins indicate that HIF- $1 \alpha$ may be directly involved in the downregulation of E-cadherin. Mechanisms include upregulation of the EMT-inducing transcription factors SNAIL (Imai et al, 2003) and TWIST (Yang et al, 2008), as well as the induction of LOX (Erler et al, 2006). So far, numerous studies have already described a downregulation of E-cadherin in cervical cancer (Vessey et al, 1995; Jeffers et al, 1997; Narayan et al, 2003; Faleiro-Rodrigues and Lopes, 2004; van de Putte et al, 2004; Rodriguez-Sastre et al, 2005; Dursun et al, 2007; Caberg et al, 2008; Lee et al, 2008). In fact, a progressive downregulation of this protein has even been observed in cervical dysplasia (de Boer et al, 1999). As cancers of the uterine cervix are the tumour entity for which the best evidence for hypoxia-associated malignant progression exists, this study has examined the question of whether correlations between the directly measured oxygenation status (Eppendorf $\mathrm{pO}_{2}$ histography), the expression of hypoxiaassociated marker proteins (GLUT-1, CA IX) and the expression of E-cadherin can be identified.

\section{MATERIALS AND METHODS}

Patients. All of the patients in this study were part of a prospective clinical trial for the evaluation of the significance of tumour oxygenation in primary, locally advanced carcinomas of the uterine cervix at the Department of Obstetrics and Gynaecology, University Medical Centre, Leipzig, Germany. The study design was approved by the local Medical Ethics Committee, with patients giving informed written consent before being enrolled. All 48 patients with squamous cell carcinomas (SCCs) for whom biopsy specimens of the oxygen measurement tracks were available were included in this study (for patient and tumour characteristics see Table 1).

Tumour oxygen tension measurements. Tumour $\mathrm{pO}_{2}$ was measured pretherapeutically with the computerised Eppendorf $\mathrm{pO}_{2}$ histography system (Eppendorf, Hamburg, Germany), using a protocol that has been described in detail (Höckel et al, 1991). Briefly, $\mathrm{pO}_{2}$ readings were performed in conscious patients along linear electrode tracks, first in the subcutaneous fat of the mons pubis followed by cervical measurements at the 12 and 6 o'clock sites in macroscopically vital tumour tissue. Within the tumour tissue, up to $35 \mathrm{pO}_{2}$ measurements were made along each electrode track (70 readings in total), starting at a tissue depth of approx. $5 \mathrm{~mm}$. The individual $\mathrm{pO}_{2}$ measurement points were situated $0.7 \mathrm{~mm}$ apart, resulting in an overall measurement track length of $25 \mathrm{~mm}$. Immediately after $\mathrm{pO}_{2}$ measurements, needle core biopsies (obtained using Biopty; Radioplast, Uppsala, Sweden) of $2 \mathrm{~mm}$ in diameter and $20 \mathrm{~mm}$ in length were taken
Table 1. Patient and tumour characteristics at the time of pretherapeutic $\mathrm{pO}_{2}$ measurements

\begin{tabular}{|l|c|r|l|}
\hline & No. of patients & $\%$ & Range \\
\hline FIGO stage & & & \\
\hline I B1 & 5 & 10.4 & \\
I B2 & 4 & 8.3 & \\
II A & 2 & 4.2 & \\
II B & 8 & 16.7 & \\
III B & 19 & 39.6 & \\
IV A & 7 & 14.6 & \\
IV B & 3 & 6.3 & \\
\hline Grade & & & \\
\hline 1 & 4 & 8.3 & \\
2 & 33 & 68.8 & \\
3 & 11 & 22.9 & \\
\hline Largest tumour diameter (mm) $^{\text {a }}$ & & & $20-120$ \\
\hline$<60$ & 22 & 45.8 & \\
$\geqslant 60$ & 26 & 54.2 & \\
\hline Patient age (yr) & & & $29-73$ \\
\hline$<50$ & 21 & 43.8 & \\
$\geqslant 50$ & 27 & 56.3 & \\
\hline cHb (g/dl) $^{\text {a }}$ & 24 & 50 & \\
\hline$<13.5$ & 24 & 50 & \\
\hline$\geqslant 13.5$ & & & \\
\hline
\end{tabular}

a The median value was used as the cut-off point between the two groups. Sums of rounded percentages may not add to exactly 100 per cent.

from those tumour areas from which $\mathrm{pO}_{2}$ readings had been obtained. Intravaginal temperature, arterial blood pressure, heart rate, haemoglobin concentration, haematocrit, and arterial oxyhaemoglobin saturation were monitored at the time when $\mathrm{pO}_{2}$ readings were taken. The pretherapeutic $\mathrm{pO}_{2}$ measurements were recorded 1-5 days before oncological treatment. The oxygenation status was described quantitatively by the parameters mean $\mathrm{pO}_{2}$, median $\mathrm{pO}_{2}$ and the percentage of $\mathrm{pO}_{2}$ values below 2.5, 5 and $10 \mathrm{mmHg}$ (i.e., 'hypoxic fractions', $\mathrm{HF} 2.5$, HF 5 and $\mathrm{HF} \mathrm{10,}$ respectively).

Immunohistochemistry and morphometry. Histologic slides were prepared from the paraffin blocks and dried overnight at $37^{\circ} \mathrm{C}$. On the next day, specimens were dewaxed in xylene and then rehydrated in a descending alcohol series. Retrieval of antigenic binding sites was performed by heating specimens in 10/1 mM Tris/EDTA buffer ( $\mathrm{pH}$ 9.0) in a steamer (Braun FS 10, Braun, Kronberg, Germany) for $40 \mathrm{~min}$. Primary antibodies against E-cadherin, GLUT-1 and CD34 were obtained from DAKO (DAKO, Hamburg, Germany, cat.-no. M3612, A3536 and M7165, respectively), and antibodies against CA IX and Ki67 were obtained from Abcam (Abcam, Cambridge, UK, cat.-no. ab15086 and ab16667). Detection of the first antigen, CD34, was carried out using overnight incubation with the primary antibody at $4{ }^{\circ} \mathrm{C}$, appropriate biotinylated secondary antibodies followed by a standard streptavidin/biotin/horseradish peroxidase reagent and colour development using DAB (brown reaction product; DAKO Duet kit). Detection of the second target protein (E-cadherin, GLUT-1 or CA IX) was preceded by a second round of antigen retrieval to prevent cross-reactions with the first antigen and to enhance unmasking efficiency. After primary antibody incubation for $1 \mathrm{~h}$ at $37^{\circ}$, the micropolymer-based, biotin-free Vector Impress detection system was applied and Vector VIP (purple reaction 
product) was used as the peroxidase substrate (Vector Laboratories, Burlingame, CA, USA). Negative control specimens were incubated in PBS without the primary antibody under the same conditions. A tumour specimen with a known strong expression of each antigen was run as a positive control with every staining batch. Slides were dehydrated in an ascending alcohol series, and covered with a coverslip using Eukitt mounting medium (Riedel-de Haen, Seelze, Germany). Digital images of the specimens were acquired with a Zeiss AxioImager microscope and AxioCam HRc using the software AxioVision (Zeiss, Oberkochen, Germany). Diffusion distances were measured in 2 to 15 areas in each biopsy using measure tools in AxioVision.

Quantitative assessment of antigen expression. Only immunostaining compatible with the known biological function and corresponding subcellular localisation of each antigen was considered as being evaluable as marker expression: membranous staining in the case of E-cadherin, GLUT-1 and CAIX, and nuclear staining for Ki67. A semiquantitative scoring system was used. For E-cadherin, score 0 , negative; score 1 , partially negative; score 2 , all cells positive. For GLUT-1, CA IX and Ki67, score 0, no staining ('absent'); score $1,<10 \%$ positive ('weak'); score $2,11 \%$ to $50 \%$ positive ('moderate'); and score $3,>50 \%$ positive ('strong'). Because of limited amounts of tissue, only 45 (of 48) biopsies stained for CA IX and 47 (of 48) stained for Ki67 could be analysed.

Protein extraction, western blotting and band densitometry. Whole-cell protein extracts (including membrane proteins) were generated using a FastPrep-24 tissue homogeniser (MP Biomedicals, Solon, OH, USA). Cryospecimens were placed in $2.0 \mathrm{ml}$ of impact-resistant tubes containing $250 \mu \mathrm{l}$ of RIPA buffer (C-C-pro, Oberdorla, Germany) supplemented with $1 \mathrm{mM}$ fresh DTT and Lysing Matrix D (i.e., $1.4 \mathrm{~mm}$ ceramic spheres, MP Biomedicals), homogenised for $10-20 \mathrm{sec}$, incubated on ice for $30 \mathrm{~min}$ and centrifuged at $15000 \mathrm{U} / \mathrm{min}$ for $30 \mathrm{~min}$ at $4{ }^{\circ} \mathrm{C}$. An aliquot of the supernatant was used for the determination of the protein concentration using Bradford's reagent (Roti-Quant, Roth, Karlsruhe, Germany), BSA as a protein standard (Sigma-Aldrich, St Louis, MO, USA) and a Tecan F200 microplate reader equipped with a 595-nm filter (Tecan, Crailsheim, Germany). A measure of $50 \mu \mathrm{g}$ of protein was separated by SDS-PAGE using a Bio-Rad Mini-Protean 3 electrophoresis chamber and transferred to PVDFmembranes using a Bio-Rad transblot SD transfer cell (Bio-Rad, Hercules, CA, USA). The primary antibody against human E-cadherin (DAKO M3612), identical to the one used for IHC, was incubated overnight at $4{ }^{\circ} \mathrm{C}$ and detected using an HRPconjugated secondary antibody (NXA931, GE Healthcare, Waukesha, WI, USA). To control for equal protein loading, membranes were stripped and reprobed with antibodies against $\beta$ actin (sc-1616r, Santa Cruz Biotechnology, Santa Cruz, CA, USA) and appropriate anti-rabbit secondary antibodies (sc-2054, Santa Cruz). Bands were developed using ECL reagent (GE Healthcare) and images were captured with a CCD camera (Fujifilm LAS-3000, Fujifilm, Tokyo, Japan). Molecular-weight determination was carried out using the Fujifilm Multigauge software and the Bio-Rad Precision Plus Dual Colour Standards, which were run on each gel.

Statistical analysis. All of the statistical tests were performed using SPSS (Version 20, IBM, Armonk, NY, USA). The significance level was set at $\alpha=5 \%$ for all comparisons. Linear correlations between two parameters were described by Spearman's rank correlation coefficient $(\rho)$. Two-sided Mann-Whitney $U$ tests and KruskalWallis tests were used for comparison of categorised variables.

Oxygenation status was correlated with FIGO stages and with tumour size, which was dichotomized using the median tumour diameter $(60 \mathrm{~mm})$ as the cut-off value. Diagrams were generated using SigmaPlot 11.

\section{RESULTS}

Prevalence of E-cadherin in SCC of the uterine cervix. On performing immunohistochemistry (IHC), the majority (52\%) of 48 squamous cell carcinomas of the uterine cervix showed strong membranous E-cadherin expression in all tumour cells. In all, 38\% of SCCs showed a partial loss (ranging from no more than focal areas of absence of the antigen to its expression in only selected groups of cells). In the remaining 10\%, E-cadherin was not detectable at all. Conversely, 9 of 37 (24\%) specimens available for western blot (WB) analyses of E-cadherin expression were negative, whereas the remaining showed $120-\mathrm{kDa}$ bands of different intensities (Supplementary Figure S1). A significant positive correlation between both assays was found $(P=0.01)$, but individual cases showed significant disagreement; for example, one tumour showed E-cadherin expression in all cells in IHC but was tested negative using WB. Owing to the lower sensitivity of the WB assay, all further results refer to the IHC data.

E-cadherin and $\mathrm{pO}_{2}$-histography data. The Kruskal-Wallis Test yielded no significant differences in the values of any of the oxygenation status parameters (mean $\mathrm{pO}_{2}$, median $\mathrm{pO}_{2}, \mathrm{HF} 2.5$, HF 5, and HF 10) between the three categories of E-Cadherin expression in the IHC analysis (i.e., the presence of the antigen in no, some or all cancer cells within the tumour biopsy). Boxplots depicting these data are presented in Figure 1.

E-cadherin and levels of GLUT-1, CA IX and Ki67. All tumours showed a varying degree of GLUT-1 expression, whereas one-third were negative for CA IX (see Table 2). Higher levels of E-cadherin were correlated with higher expression scores of the endogenous hypoxia-related markers GLUT-1 $(\rho=0.51, P<0.001)$ and CA IX $(\rho=0.33, P=0.03)$. Expression levels of GLUT-1 and CA IX were also correlated with each other $(\rho=0.48, P=0.001)$. Correlations with proliferation (Ki67) were not found.

Expression patterns and interrelationships of E-cadherin, GLUT-1, CA IX and Ki67. Focal downregulation of E-cadherin in GLUT-1- or CA IX-positive areas, as expected from the concept of hypoxia-induced EMT, was not observed. In subregions of most biopsies (41 of 48), expression of GLUT-1 was found to increase beyond an average distance of $76 \mu \mathrm{m}$ from the nearest microvessel

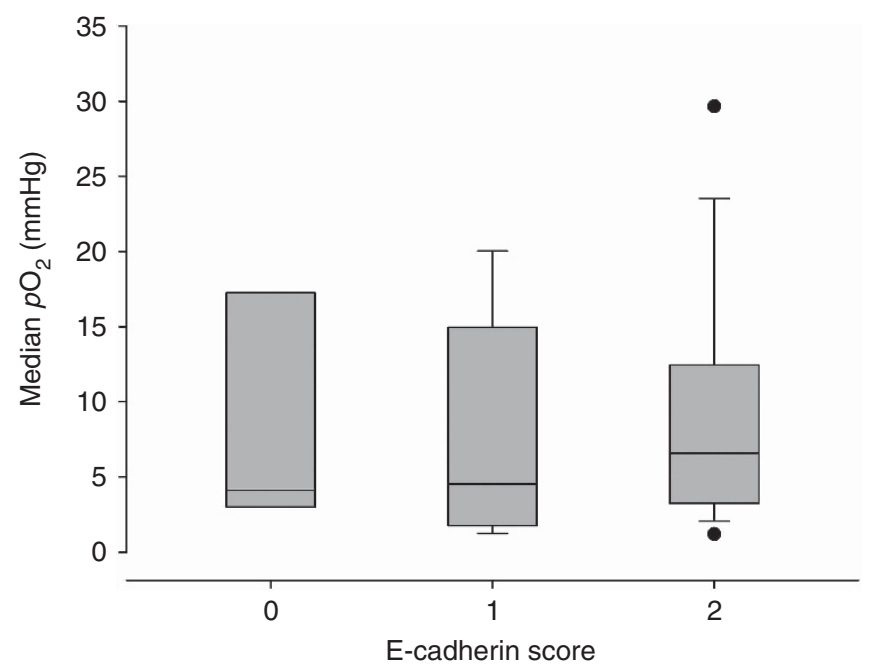

Figure 1. No correlation between E-cadherin expression and the oxygenation status of cervix cancers. Boxplots show equal distributions of median $\mathrm{pO}_{2}$ values in the three categories of E-cadherin expression. Similar distributions were found for mean $\mathrm{pO}_{2}$ values and hypoxic fractions (see text for details). 
(CD34 expression), as evaluated by multiple manual measurements in each double-stained section (see Figure $2 \mathrm{~A}$ for an example). Of 30 CA IX-positive biopsies, 23 showed increased antigen expression at a mean distance of $83 \mu \mathrm{m}$ from the nearest microvessel. In the remaining seven positive tumours, a relationship between CA IX expression and diffusion distances could not be identified. Conversely, no spatial relationship between focal areas of E-cadherin downregulation and microvessels was observed in any of the positive biopsies (Figure 2B). E-cadherin expression was also independent of the local growth pattern. To illustrate this observation, Figure $3 \mathrm{~A}$ shows an example of strongly E-cadherin-positive cells that exhibit a reticular pattern of infiltration. Conversely, Figure 3B shows compact and coherent tumour cell aggregates that completely lack the expression of E-cadherin.

Other findings. No correlation was found between clinical tumour size and the oxygenation status when both parameters

\begin{tabular}{|c|c|c|c|c|}
\hline & GLUT-1 & & CA IX & \\
\hline Score & number of patients & $\%$ & number of patients & $\%$ \\
\hline 0 & 0 & 0 & 15 & 33.3 \\
\hline 1 & 11 & 22.9 & 10 & 22.2 \\
\hline 2 & 13 & 27.1 & 16 & 35.6 \\
\hline 3 & 24 & 50.0 & 4 & 8.9 \\
\hline Total & 48 & 100.0 & 45 & 100.0 \\
\hline
\end{tabular}

were tested as continuous variables. When tumour size was dichotomized using the median value as the cut-off, larger tumours showed a trend towards a slightly poorer oxygenation status, but only regarding the mean $\mathrm{pO}_{2}(P=0.054)$. Higher FIGO stages, however, did not show a poorer oxygenation status.

\section{DISCUSSION}

Beginning with the experiments of Coman (1944), who was able to show almost 70 years ago that scrapings obtained from squamous cell carcinomas of the uterine cervix exhibit a reduced intercellular cohesion, numerous studies have investigated the importance of loosened cell-cell contacts for the invasiveness of cancers of this and other tumour types. It had been recognised even earlier that extracellular $\mathrm{Ca}^{2+}$ levels are important for cell-to-cell coherence, and it is now established that the (calcium-dependent) homotypic interaction of the extracellular domains of E-cadherin molecules, located in adherens junctions, is crucial for the mechanical stability of intercellular contacts (Perez-Moreno et al, 2003). Possible mechanisms mediating a specific downregulation of these structures in hypoxic tumour areas are of great interest, as numerous studies have demonstrated a significant prognostic importance of hypoxia and hypoxia-associated proteome changes. Our study is the first to investigate this question in SCCs of the uterine cervix. Our results clearly demonstrate that hypoxic tumours or tumours with a high expression of hypoxia-associated proteins do not show downregulation of E-cadherin or other characteristic signs of EMT. On the contrary, we have even

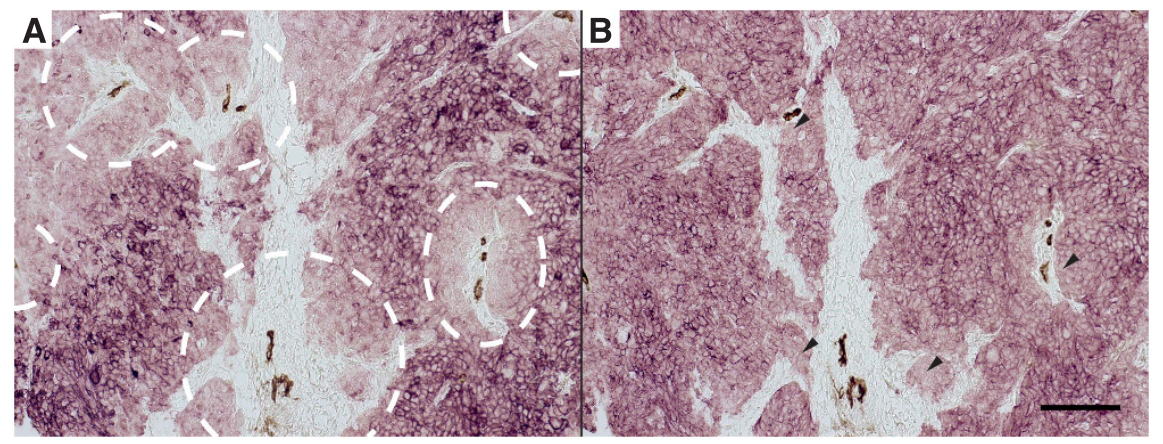

Figure 2. Expression pattern of GLUT-1, but not E-cadherin, is related to diffusion gradients in cancers of the uterine cervix. GLUT-1 expression (purple) shows a gradual increase with increasing distance from the microvessels (brown) in panel (A). Circular areas of low GLUT-1 expression (marked by white dashed lines) can be identified around selected microvessels. No such pattern is evident for E-cadherin (purple) in panel (B). $200 \times$ magnification, scale bar in $\mathbf{B}=100 \mu \mathrm{m}$.
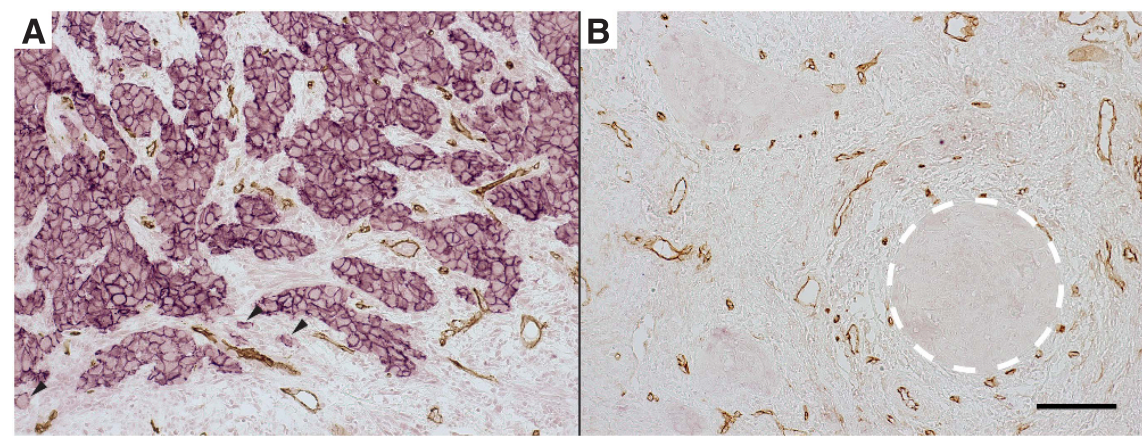

Figure 3. Expression of E-cadherin is not related to the microregional growth pattern of cancers of the uterine cervix. A reticular, highly branched pattern of invasion, including apparently scattered single cells (arrowheads), can be observed in tumours with strong membranous E-cadherin expression (A). Conversely, compact, spheroid-like configurations of tumour cell clusters (demarcated by a white dashed line) can be observed in tumours completely negative for E-cadherin (B). $200 \times$ magnification, scale bar in $\mathbf{B}=100 \mu \mathrm{m}$. 
observed significant positive correlations between the expression of the hypoxia-associated proteins (GLUT-1 and CA IX) and E-cadherin. Such a correlation would seem plausible if a reduced E-cadherin expression were to be accompanied by increased cell scattering, which, in turn, would lead to the 'reoxygenation' of tumour cells upon their infiltration into the microvessel-carrying and thus 'oxygen-providing' tumour stroma. This possibility was, however, excluded in this study, as in terms of E-cadherin expression no systematic differences between highly fragmented tumour cell clusters and isolated tumour cells on the one hand and coherent tumour cell aggregates on the other could be identified. As shown in Figure 3, examples both of tumour areas in the first category (scattered phenotype), which consistently showed strong expression of E-cadherin, and areas in the second category (cohesive phenotype), which completely lacked the expression of this antigen, have been found. These observations confirm earlier findings in cancers of the uterine cervix (Jeffers et al, 1997) and breast cancer (Colpaert et al, 2003), which also did not show an impact of E-cadherin expression on the invasion pattern. With regard to the positive correlations between E-cadherin and hypoxia-related markers, we currently have no biologically plausible explanation for this finding, but would assume that it is unlikely that these findings are the result of technical shortcomings of our immunohistochemical protocols, as these were already validated extensively in prior studies (e.g., Mayer et al (2008)).

Jeffers et al (1997) also reported E-cadherin expression in all 20 tumours investigated in their study, i.e., even more than the $90 \%$ positive tumours identified in our work (in the IHC assay). Nevertheless, both studies stand out among the literature, which commonly reported much lower E-cadherin levels. For example, the largest study to date, the work of van de Putte et al (2004), which comprised 219 patients, reported a high expression of E-Cadherin (in more than $50 \%$ of the tumour cells) being restricted to only $10 \%$ of the patients analysed, whereas our own results show $100 \%$ positive tumour cells in more than half of our patient cohort. Furthermore, as opposed to our study, which found only 10\% of patients to have completely negative tumours, relatively high rates of negative tumours ( 34 to $100 \%$ ) have been reported in the majority of prior studies (e.g., Ancuta et al (2009); FaleiroRodrigues and Lopes (2004); Lee et al (2008); Rodriguez-Sastre et al (2005)). These differences may partly be explained by the use of different tissue fixation protocols, primary antibody clones and detection systems or a combination of these factors. Indeed, the use of the purple peroxidase substrate VIP for the detection of E-cadherin - which in our study was performed to enable double staining with CD34 - resulted in much stronger (yet decisively specific) staining results than $\mathrm{DAB}$, as tested during the pilot phase of our study (data not shown). Particular incongruities exist between our study and the report from Lee et al (2008), who studied SCC of the uterine cervix using immunofluorescence detection of E-cadherin and vimentin in specimens, which, according to the interpretation of these authors, represented an ascending order of local spread (i.e., normal squamous epithelium, superficial tumour tissue, tumour cell nests in the parametrium and tumour cells in pelvic lymph nodes). They found a progressive decrease of E-cadherin and an opposed induction of vimentin, suggesting bona fide EMT in 10 out of 10 patients analysed. Interestingly, their results agree with our data in one important aspect: E-cadherin downregulation obviously did not necessarily lead to single-cell scattering, as is evident from the E-cadherinnegative, but still compact, tumour cell nodule found in the parametrium in the study of Lee et al (see Figure 1A in Lee et al (2008) compared to Figure 3B of this study). Nevertheless, we are currently unable to explain the substantial differences between these two studies.

Detection of E-Cadherin using western blotting of tissue extracts confirmed the finding of maintained E-cadherin expression in the vast majority of tumours. However, in our hands, western blots turned out to be significantly less sensitive than IHC, which is why we relied on data from the latter assay for all further analyses. The inability to check for the amount of stromal tissue present in the portion of the biopsy lysed for protein extraction is a significant drawback of this method and may well be decisive for the quantitative differences between the results of these two methods. We are aware of the fact that the finding of preserved E-cadherin expression in the cell membrane of the majority of cancer cells investigated in our study does not necessarily imply that these molecules are fully functional. However, this aspect was not investigated in our work, as the hypothesis explicitly implied a downregulation of protein abundance. This topic may, however, be an interesting subject for further studies. E-Cadherin expression levels were also not correlated with the proliferation rate, but knowledge of the expression pattern of this antigen in consecutive sections enabled us to ascertain that our analyses were carried out in proliferating, and hence vital, areas of the tumours.

In keeping with the results of our prior studies in the same tumour entity (Mayer et al, 2005a, b), we again did not observe correlations between endogenous hypoxia-related markers GLUT1 and CA IX and any parameter of the oxygenation status in this completely different and more recent cohort of patients. As our protocol specified biopsy acquisition from tumour microregions identical to the areas in which oxygenation measurements had been made, the important influence of tumour heterogeneity on the expression of hypoxia-related markers has already been minimised by our approach. However, tumour heterogeneity alone is probably not sufficient to explain these discrepancies, as even the elaborate IHC analysis protocol of systematically investigating 3 levels of two different biopsies used in the study of Iakovlev et al (2007) did not result in the identification of correlations between the oxygenation status and the expression of CA IX. Correlations between HIF-dependent markers and oxygen microsensor measurements may also be distorted by the occurrence of tumour necroses, which are severely hypoxic using needle electrode measurements (Lyng et al, 1997; Jenkins et al, 2000) but do not necessarily go hand-in-hand with extensive expression of HIFrelated markers in the same specimens. The influence of this factor is also expected to be rather modest in our study, as $\mathrm{pO}_{2}$ measurements and corresponding biopsies were excluded from analyses in cases in which histopathological examination showed widespread tumour necrosis. Conversely, it has long been known that the expression levels of both HIF- $1 \alpha$ and its target genes GLUT-1 and CA IX, as investigated in this study, are influenced by a variety of factors other than hypoxia, such as glucose deprivation (Boado and Pardridge, 2002; Kwon and Lee, 2005), acidosis (Mekhail et al, 2004), oncogenes (e.g., c-MYC (Osthus et al, 2000)) and oncogene-associated signalling pathways (e.g., PI3K/AKT (Kaluz et al, 2002)). These significant hypoxia-independent influences have been summarised in a review (Mayer et al, 2006). In addition, recent research has demonstrated a role for reactive oxygen species and NO in stabilizing HIF- $1 \alpha$ (Fong and Takeda, 2008; Dewhirst, 2009).

Beyond mere quantitative correlations between E-cadherin, GLUT-1 and CA IX expression levels, we were able to carry out systematic computer-aided classifications of antigen expression patterns with regard to the local diffusion geometry (by measuring distances from marker-positive cells to the nearest microvessel), as sections of all three antigens had each been double-stained for the marker CD34 (microvascular endothelium). Results of this analysis showed that most of the (antigen-positive) tumours exhibited the expected pattern of expression of GLUT and CA IX beyond a mean distance of approx. $80 \mu \mathrm{m}$ (76 $\mu \mathrm{m}$ for GLUT-1 and $83 \mu \mathrm{m}$ for CA IX) from the nearest microvessels in subregions of the biopsies. In contrast, such a constellation could not be identified for E-cadherin, which was typically expressed both in the immediate 
vicinity of blood vessels and areas further down the oxygen diffusion gradients. The absence of any systematic spatial relationship between E-cadherin and the hypoxia-associated proteins clearly contradicts the model of a hypoxia-mediated downregulation of E-cadherin in cancers of the uterine cervix.

The results obtained in this study indicate that downregulation of E-cadherin in cancers of the uterine cervix does not show associations with the 'physical' oxygenation status of the tissue or with the locoregional expression levels of hypoxia-related markers. Hence, other mechanisms leading to E-cadherin downregulation may predominate in this tumour entity. However, the possibility that the general importance of E-cadherin downregulation (and of the associated EMT) may be smaller in SCCs of the uterine cervix than in other tumour entities should also be considered. It is well known that alternative patterns of tumour invasion exist (Friedl and Wolf, 2003) and, according to one study (Wicki et al, 2006), a collective migration pattern may in fact predominate in SCCs of the uterine cervix, consistent with our finding of widespread E-cadherin expression in this entity. In addition, in a recent study, we have identified recurrent histological signs indicating an active role of the tumour stroma in mediating invasiveness in the form of cell collectives in this tumour entity (Mayer et al, 2011). Indeed, prior experimental findings have directly visualised how fibroblasts may enable the invasion of cancer cells derived from SCCs (e.g., Gaggioli et al, (2007)). Future work is needed to clarify to what extent hypoxia-mediated mechanisms may have a role in the context of these alternative types of cancer invasion.

\section{ACKNOWLEDGEMENTS}

The authors thank Dr. Debra Kelleher for her valuable editorial help during preparation of this manuscript. This work was supported by a grant from the Deutsche Krebshilfe (no. 106758).

\section{CONFLICT OF INTEREST}

The authors declare no conflict of interest.

\section{REFERENCES}

Ancuta E, Ancuta C, Cozma LG, Iordache C, Anghelache-Lupascu I, Anton E, Carasevici E, Chirieac R (2009) Tumor biomarkers in cervical cancer: focus on Ki-67 proliferation factor and E-cadherin expression. Rom J Morphol Embryol 50: 413-418.

Boado RJ, Pardridge WM (2002) Glucose deprivation and hypoxia increase the expression of the GLUT1 glucose transporter via a specific mRNA cisacting regulatory element. J Neurochem 80: 552-554.

Brizel DM, Scully SP, Harrelson JM, Layfield LJ, Bean JM, Prosnitz LR, Dewhirst MW (1996) Tumor oxygenation predicts for the likelihood of distant metastases in human soft tissue sarcoma. Cancer Res 56: 941-943.

Caberg JH, Hubert PM, Begon DY, Herfs MF, Roncarati PJ, Boniver JJ, Delvenne PO (2008) Silencing of E7 oncogene restores functional E-cadherin expression in human papillomavirus 16-transformed keratinocytes. Carcinogenesis 29: 1441-1447.

Colpaert CG, Vermeulen PB, van Beest P, Soubry A, Goovaerts G, Dirix LY, Harris AL, van Marck EA (2003) Cutaneous breast cancer deposits show distinct growth patterns with different degrees of angiogenesis, hypoxia and fibrin deposition. Histopathology 42: 530-540.

Coman DR (1944) Decreased mutual adhesiveness, a property of cells from squamous cell carcinomas. Cancer Res 4: 625-629.

de Boer CJ, van Dorst E, van Krieken H, Jansen-van Rhijn CM, Warnaar SO, Fleuren GJ, Litvinov SV (1999) Changing roles of cadherins and catenins during progression of squamous intraepithelial lesions in the uterine cervix. Am J Pathol 155: 505-515.
Dewhirst MW (2009) Relationships between cycling hypoxia, HIF-1, angiogenesis and oxidative stress. Radiat Res 172: 653-665.

Dursun P, Yuce K, Usubutun A, Ayhan A (2007) Loss of epithelium cadherin expression is associated with reduced overall survival and disease-free survival in early-stage squamous cell cervical carcinoma. Int J Gynecol Cancer 17: 843-850.

Erler JT, Bennewith KL, Nicolau M, Dornhöfer N, Kong C, Le QT, Chi JT, Jeffrey SS, Giaccia AJ (2006) Lysyl oxidase is essential for hypoxia-induced metastasis. Nature 440: 1222-1226.

Faleiro-Rodrigues C, Lopes C (2004) E-cadherin, CD44 and CD44v6 in squamous intraepithelial lesions and invasive carcinomas of the uterine cervix: an immunohistochemical study. Pathobiology 71: 329-336.

Fong GH, Takeda K (2008) Role and regulation of prolyl hydroxylase domain proteins. Cell Death Differ 15: 635-641.

Friedl P, Wolf K (2003) Tumour-cell invasion and migration: diversity and escape mechanisms. Nat Rev Cancer 3: 362-374.

Gaggioli C, Hooper S, Hidalgo-Carcedo C, Grosse R, Marshall JF, Harrington K, Sahai E (2007) Fibroblast-led collective invasion of carcinoma cells with differing roles for RhoGTPases in leading and following cells. Nat Cell Biol 9: $1392-1400$.

Höckel M, Knoop C, Schlenger K, Vorndran B, Baussmann E, Mitze M, Knapstein PG, Vaupel P (1993) Intratumoral $\mathrm{pO}_{2}$ predicts survival in advanced cancer of the uterine cervix. Radiother Oncol 26: 45-50.

Höckel M, Schlenger K, Aral B, Mitze M, Schäffer U, Vaupel P (1996) Association between tumor hypoxia and malignant progression in advanced cancer of the uterine cervix. Cancer Res 56: 4509-4515.

Höckel M, Schlenger K, Höckel S, Aral B, Schäffer U, Vaupel P (1998) Tumor hypoxia in pelvic recurrences of cervical cancer. Int J Cancer 79: 365-369.

Höckel M, Schlenger K, Knoop C, Vaupel P (1991) Oxygenation of carcinomas of the uterine cervix: evaluation by computerized $\mathrm{O}_{2}$ tension measurements. Cancer Res 51: 6098-6102.

Iakovlev VV, Pintilie M, Morrison A, Fyles AW, Hill RP, Hedley DW (2007) Effect of distributional heterogeneity on the analysis of tumor hypoxia based on carbonic anhydrase IX. Lab Invest 87: 1206-1217.

Imai $\mathrm{T}$, Horiuchi $\mathrm{A}$, Wang $\mathrm{C}$, Oka $\mathrm{K}$, Ohira S, Nikaido T, Konishi I (2003) Hypoxia attenuates the expression of E-cadherin via up-regulation of SNAIL in ovarian carcinoma cells. Am J Pathol 163: 1437-1447.

Jeffers MD, Paxton J, Bolger B, Richmond JA, Kennedy JH, McNicol AM (1997) E-cadherin and integrin cell adhesion molecule expression in invasive and in situ carcinoma of the cervix. Gynecol Oncol 64: 481-486.

Jenkins WT, Evans SM, Koch CJ (2000) Hypoxia and necrosis in rat $9 \mathrm{~L}$ glioma and Morris 7777 hepatoma tumors: comparative measurements using EF5 binding and the Eppendorf needle electrode. Int J Radiat Oncol Biol Phys 46: 1005-1017.

Kaluz S, Kaluzova M, Chrastina A, Olive PL, Pastorekova S, Pastorek J, Lerman MI, Stanbridge EJ (2002) Lowered oxygen tension induces expression of the hypoxia marker MN/carbonic anhydrase IX in the absence of hypoxia-inducible factor $1 \alpha$ stabilization: a role for phosphatidylinositol 3'-kinase. Cancer Res 62: 4469-4477.

Kwon SJ, Lee YJ (2005) Effect of low glutamine/glucose on hypoxia-induced elevation of hypoxia-inducible factor- $1 \alpha$ in human pancreatic cancer MiaPaCa-2 and human prostatic cancer DU-145 cells. Clin Cancer Res 11: 4694-4670.

Lee MY, Chou CY, Tang MJ, Shen MR (2008) Epithelial-mesenchymal transition in cervical cancer: correlation with tumor progression, epidermal growth factor receptor overexpression, and snail up-regulation. Clin Cancer Res 14: 4743-4750.

Lyng H, Sundfor K, Rofstad EK (1997) Oxygen tension in human tumours measured with polarographic needle electrodes and its relationship to vascular density, necrosis and hypoxia. Radiother Oncol 44: 163-169.

Mayer A, Höckel M, Horn LC, Schmidberger H, Vaupel P (2011) GLUT-1 staining of squamous cell carcinomas of the uterine cervix identifies a novel element of invasion. Int J Oncol 38: 145-150.

Mayer A, Höckel M, Vaupel P (2005a) Carbonic anhydrase IX expression and tumor oxygenation status do not correlate at the microregional level in locally advanced cancers of the uterine cervix. Clin Cancer Res 11: $7220-7225$.

Mayer A, Höckel M, Vaupel P (2006) Endogenous hypoxia markers in locally advanced cancers of the uterine cervix: reality or wishful thinking? Strahlenther Onkol 182: 501-510. 
Mayer A, Höckel M, Wree A, Leo C, Horn LC, Vaupel P (2008) Lack of hypoxic response in uterine leiomyomas despite severe tissue hypoxia. Cancer Res 68: 4719-4726.

Mayer A, Höckel M, Wree A, Vaupel P (2005b) Microregional expression of glucose transporter-1 and oxygenation status: lack of correlation in locally advanced cervical cancers. Clin Cancer Res 11: 2768-2773.

Mekhail K, Gunaratnam L, Bonicalzi ME, Lee S (2004) HIF activation by pH-dependent nucleolar sequestration of VHL. Nat Cell Biol 6: 642-647.

Narayan G, Arias-Pulido H, Koul S, Vargas H, Zhang FF, Villella J, Schneider A, Terry MB, Mansukhani M, Murty VV (2003) Frequent promoter methylation of CDH1, DAPK, RARB, and HIC1 genes in carcinoma of cervix uteri: its relationship to clinical outcome. Mol Cancer 2: 24 .

Nordsmark M, Bentzen SM, Rudat V, Brizel D, Lartigau E, Stadler P, Becker A, Adam M, Molls M, Dunst J, Terris DJ, Overgaard J (2005) Prognostic value of tumor oxygenation in 397 head and neck tumors after primary radiation therapy. An international multi-center study. Radiother Oncol 77: $18-24$.

Osthus RC, Shim H, Kim S, Li Q, Reddy R, Mukherjee M, Xu Y, Wonsey D, Lee LA, Dang CV (2000) Deregulation of glucose transporter 1 and glycolytic gene expression by c-Myc. J Biol Chem 275: 21797-21800.

Perez-Moreno M, Jamora C, Fuchs E (2003) Sticky business: orchestrating cellular signals at adherens junctions. Cell 112: 535-548.

Rodriguez-Sastre MA, Gonzalez-Maya L, Delgado R, Lizano M, Tsubaki G, Mohar A, Garcia-Carranca A (2005) Abnormal distribution of E-cadherin and $B$-catenin in different histologic types of cancer of the uterine cervix Gynecol Oncol 97: 330-336.

Semenza GL (2012) Hypoxia-inducible factors: mediators of cancer progression and targets for cancer therapy. Trends Pharmacol Sci 33: 207-214. van de Putte G, Kristensen GB, Baekelandt M, Lie AK, Holm R (2004) E-cadherin and catenins in early squamous cervical carcinoma. Gynecol Oncol 94: 521-527.

Vaupel P, Mayer A (2007) Hypoxia in cancer: significance and impact on clinical outcome. Cancer Metastasis Rev 26: 225-239.

Vaupel P, Mayer A, Höckel M (2004) Tumor hypoxia and malignant progression. Methods Enzymol 381: 335-354.

Vessey CJ, Wilding J, Folarin N, Hirano S, Takeichi M, Soutter P, Stamp GW, Pignatelli M (1995) Altered expression and function of E-cadherin in cervical intraepithelial neoplasia and invasive squamous cell carcinoma. J Pathol 176: 151-159.

Wang GL, Jiang BH, Rue EA, Semenza GL (1995) Hypoxia-inducible factor 1 is a basic-helix-loop-helix-PAS heterodimer regulated by cellular $\mathrm{O}_{2}$ tension. Proc Natl Acad Sci USA 92: 5510-5514.

Wenger RH (2002) Cellular adaptation to hypoxia: $\mathrm{O}_{2}$-sensing protein hydroxylases, hypoxia-inducible transcription factors, and $\mathrm{O}_{2}$-regulated gene expression. FASEB J 16: 1151-1162.

Wicki A, Lehembre F, Wick N, Hantusch B, Kerjaschki D, Christofori G (2006) Tumor invasion in the absence of epithelial-mesenchymal transition: podoplanin-mediated remodeling of the actin cytoskeleton. Cancer Cell 9: 261-272.

Yang MH, Wu MZ, Chiou SH, Chen PM, Chang SY, Liu CJ, Teng SC, Wu KJ (2008) Direct regulation of TWIST by HIF-1alpha promotes metastasis. Nat Cell Biol 10: 295-305.

cc)(1)(2) This work is licensed under the Creative Commons (c) Attribution-NonCommercial-Share Alike 3.0 Unported License. To view a copy of this license, visit http://creativecommons. org/licenses/by-nc-sa/3.0/

Supplementary Information accompanies this paper on British Journal of Cancer website (http://www.nature.com/bjc) 